\title{
SOME FAMILIES OF ONE AND TWO-STEP ITERATIVE METHODS FOR APPROXIMATING MULTIPLE ROOTS OF NONLINEAR EQUATIONS
}

\author{
Mohamed S. M. Bahgat* and Mufida A. Nasr* \\ msmbahgat66@hotmail.com and mofeda.nasr@yahoo.com
}

Received 1/3/2018 Revised 10/3/2018 Accepted 27/4/2018

\begin{abstract}
This paper presents a new family of two-step iterative methods as a new modification to the Halley's method. Also a family of one-step iterative methods is generated using the variational iteration technique to approximate the multiple roots of nonlinear equations with unknown multiplicity $\beta$. The order of convergence is analyzed and proved that, some of the proposed methods reach the optimal order of convergence, which is higher than that of Newton's method. The outcomes for some numerical examples are given to approve the efficiency of the presented methods compared with some other known methods. Mathematics Subject Classification: 34A45, 34A45, 37N30.
\end{abstract}

Keywords: nonlinear equations; convergence analysis; multiple roots; Halley's method; efficiency index.

\section{Introduction}

Analytical methods for solving nonlinear equations are almost non-existent and therefore, it is only possible to obtain approximate numerical solutions basing on iterative methods. Solving for the multiple roots of nonlinear equations with unknown multiplicity is one of the most important problems in numerical analysis, and was the subject of many researchers (see $[1-8]$ ). Finding iterative methods for solving multiple roots of nonlinear equations efficiently and accurately is interesting and challenging in computational mathematics, especially to handle the problems of the integral transforms. These multiple roots pose difficulties when the investigated function does not change sign at even multiple roots, preclude the use of bracketing methods, and limit developing to open methods. Because the methods known to handle the case of multiple roots are few, the target of this paper is to present a family of iterative methods of higher order for finding multiple zeros of a nonlinear equations. We consider iterative methods to find a real multiple root $\alpha$ of multiplicity of nonlinear equation $f(x)=0$ with unknown multiplicity $\beta$. The multiplicity $\beta$ is the highest multiplicity, where $f:[a ; b] \subset R \rightarrow R$ is a nonlinear differential function on $[a, b]$, i.e., $f^{(i)}(\alpha)=0, \quad i=0,1, \ldots, \beta-1$ and $f^{(\beta)}(\alpha) \neq 0$. The improved Newton method $[1]$ for multiple roots is given by

$$
x_{m+1}=x_{m}-\beta f\left(x_{m}\right)\left(f^{\prime}\left(x_{m}\right)\right)^{-1}, \quad \beta>0, \quad m=0,1,2, \ldots
$$

Which starts and converges quadratically. In this work, we shall use (1) to construct our proposed methods to improve the order of convergence of iterative methods to find for multiple roots. We present higher order convergence methods basing on six evaluations of the function per iteration. In addition, the new ninth-order methods presented in this work have an efficiency index better than the fourth and sixth-order methods. The contents of the paper are summarized as follows. In 2, we describe the proposed fourth, sixth and ninth-order methods with two-step iterative and suggest some methods of

*Mathematics Department, Faculty of Science, El-Minia University, El-Minia, Egypt. 
one-step iterative basing on the variational iteration technique for finding the multiple roots of nonlinear equations. The order of convergence for each method is analyzed and proved. In 3, some numerical examples are given to demonstrate the performance of the presented methods compared with previous ones in literature. Finally conclusions of this work appear in 4 .

\section{The presented methods and analysis of convergence}

In this section, we introduce new fourth, sixth and ninth-order iterative methods for finding the real multiple roots of a nonlinear equation. These iterative methods are based on the third-order method presented recently by authors in [9-11]. We will denote these methods by Bahgat and Mufida Methods BMM1 to BMM11 as follows

BMM1: Consider the following iterative algorithm in [10],

$$
y_{m}=x_{m}-\beta f\left(x_{m}\right)\left(f^{\prime}\left(x_{m}\right)\right)^{-1}, \quad \beta>0,
$$

and

$$
y_{m}=x_{m}-(\beta-1) \frac{f^{\prime}\left(x_{m}\right)}{f^{\prime \prime}\left(x_{m}\right)},
$$

converge quadratically. By taking arithmetic mean of (2) and (3), we get

$$
y_{m}=x_{m}-\frac{1}{2}\left(\beta \frac{f\left(x_{m}\right)}{f^{\prime}\left(x_{m}\right)}+(\beta-1) \frac{f^{\prime}\left(x_{m}\right)}{f^{\prime \prime}\left(x_{m}\right)}\right),
$$

which has quadratic convergence and satisfies the following error equation,

$$
e_{m+1}=\left(\frac{c_{1}}{\beta-1}\right) e_{m}^{2}+O\left(e_{m}^{3}\right)
$$

where $c_{1}=\frac{\beta ! f^{(\beta+1)}(\alpha)}{(\beta+1) ! f^{(\beta)}(\alpha)}$. We use (4) as a predictor-corrector, then we have the following new scheme which has fourth-order convergence method is given by

$$
\begin{aligned}
& y_{m}=x_{m}-\frac{1}{2}\left(\beta\left(f^{\prime}\left(x_{m}\right) f\left(x_{m}\right)\right)^{-1}+(\beta-1)\left(f^{\prime \prime}\left(x_{m}\right) f^{\prime}\left(x_{m}\right)\right)^{-1}\right), \\
& x_{m+1}=y_{m}-\frac{1}{2}\left(\beta\left(f^{\prime}\left(y_{m}\right) f\left(y_{m}\right)\right)^{-1}+(\beta-1)\left(f^{\prime \prime}\left(y_{m}\right) f^{\prime}\left(y_{m}\right)\right)^{-1}\right),
\end{aligned}
$$

where $m \in N, \beta>1$ and $x_{0}$ is the initial guess the denominator of (5) is provided not to equal to zero.

BMM2: It is well established that the first step is the third order method which is the Halley's method [9], and the new second step is seem in (5) consequently. Then we obtain a new sixth-order convergence algorithm in the form

$$
\begin{gathered}
y_{m}=x_{m}-\frac{f\left(x_{m}\right)}{\frac{\beta+1}{2 \beta} f^{\prime}\left(x_{m}\right)-\frac{f\left(x_{m}\right) f^{\prime \prime}\left(x_{m}\right)}{2 f^{\prime}\left(x_{m}\right)}}, \quad \beta>1 \\
x_{m+1}=y_{m}-\frac{1}{2}\left(\beta \frac{f\left(y_{m}\right)}{f^{\prime}\left(y_{m}\right)}+(\beta-1) \frac{f^{\prime}\left(y_{m}\right)}{f^{\prime \prime}\left(y_{m}\right)}\right),
\end{gathered}
$$

BMM3: Let's consider the quadratically convergent scheme

$$
y_{m}=x_{m}-(\beta-1) \frac{f^{\prime}\left(x_{m}\right)}{f^{\prime \prime}\left(x_{m}\right)},
$$

and the method in $[10,12]$

$$
y_{m}=x_{m}-f\left(x_{m}\right) f^{\prime}\left(x_{m}\right)\left(\left(f^{\prime}\left(x_{m}\right)\right)^{2}-f\left(x_{m}\right) f^{\prime \prime}\left(x_{m}\right)\right)^{-1},
$$

then we get from (7) and (8)

$$
y_{m}=x_{m}-\frac{1}{2}\left((\beta-1) \frac{f^{\prime}\left(x_{m}\right)}{f^{\prime \prime}\left(x_{m}\right)}+\frac{f\left(x_{m}\right) f^{\prime}\left(x_{m}\right)}{\left(f^{\prime}\left(x_{m}\right)\right)^{2}-f\left(x_{m}\right) f^{\prime \prime}\left(x_{m}\right)}\right),
$$


which can be regarded as an arithmetic mean of two values namely, $(\beta-1) \frac{f^{\prime}\left(x_{m}\right)}{f^{\prime \prime}\left(x_{m}\right)}$ and $\frac{f\left(x_{m}\right) f^{\prime}\left(x_{m}\right)}{\left(f^{\prime}\left(x_{m}\right)\right)^{2}-f\left(x_{m}\right) f^{\prime \prime}\left(x_{m}\right)}$, which satisfy the following error equation

$$
e_{m+1}=\frac{c_{1}}{\beta(\beta-1)} e_{m}^{2}+O\left(e_{m}^{3}\right)
$$

Now, to improve the order of (9) from two to three, we introduce two free disposable parameters $k_{1}$ and $k_{2}$ in (9) and get

$$
y_{m}=x_{m}-\frac{1}{2}\left((\beta-1) \frac{k_{1} f^{\prime}\left(x_{m}\right)}{f^{\prime \prime}\left(x_{m}\right)}+\frac{k_{2} f\left(x_{m}\right) f^{\prime}\left(x_{m}\right)}{\left(f^{\prime}\left(x_{m}\right)\right)^{2}-f\left(x_{m}\right) f^{\prime \prime}\left(x_{m}\right)}\right),
$$

which satisfies the following error equation

$$
e_{m+1}=\frac{2-k_{1}-k_{2}}{2} e_{m}+\frac{(a+b+a \beta+b \beta) c_{1}}{2 \beta(\beta-1)} e_{m}^{2}+O\left(e_{m}^{3}\right)=B_{3} e_{m}+B_{4} e_{m}^{2}+O\left(e_{m}^{3}\right),
$$

Therefore, $B_{3}$ and $B_{4}$ must be equal to zero to get a cubically convergent method. Solving for $B_{3}=\frac{2-k_{1}-k_{2}}{2}=0$ and $B_{4}=\frac{(a+b+a \beta+b \beta) c_{1}}{2 \beta(\beta-1)}=0$, we get $k_{1}=\frac{\beta-1}{\beta}, k_{2}=\frac{\beta+1}{\beta}$ and $a=-b$. Hence, inserting $k_{1}$ and $k_{2}$ into (10), we obtain

$$
\begin{gathered}
y_{m}=x_{m}-\frac{1}{2 \beta}\left((\beta-1)^{2} \frac{f^{\prime}\left(x_{m}\right)}{f^{\prime \prime}\left(x_{m}\right)}+\left((\beta+1) f\left(x_{m}\right) f^{\prime}\left(x_{m}\right)\right)\left(\left(f^{\prime}\left(x_{m}\right)\right)^{2}-f\left(x_{m}\right) f^{\prime \prime}\left(x_{m}\right)\right)^{-1}\right), \\
x_{m+1}=y_{m}-\frac{1}{2 \beta}\left((\beta-1)^{2} \frac{f^{\prime}\left(y_{m}\right)}{f^{\prime \prime}\left(y_{m}\right)}+\frac{(\beta+1) f^{\prime}\left(y_{m}\right) f\left(y_{m}\right)}{\left(f^{\prime}\left(x_{m}\right)\right)^{2}-f^{\prime \prime}\left(y_{m}\right) f\left(y_{m}\right)}\right) \quad \beta>1 .
\end{gathered}
$$

is a new ninth-order method for multiple roots which requires six functional evaluations.

BMM4: The ninth-order iterative method is based on the Halley's method [9] and the new second step in (11), then the new method of order nine is given as,

$$
\begin{gathered}
y_{m}=x_{m}-\frac{f\left(x_{m}\right)}{\frac{\beta+1}{2 \beta} f^{\prime}\left(x_{m}\right)-\frac{f\left(x_{m}\right) f^{\prime \prime}\left(x_{m}\right)}{2 f^{\prime}\left(x_{m}\right)} .} . \\
x_{m+1}=y_{m}-\frac{1}{2 \beta}\left((\beta-1)^{2} \frac{f^{\prime}\left(y_{m}\right)}{f^{\prime \prime}\left(y_{m}\right)}+\frac{(\beta+1) f\left(y_{m}\right) f^{\prime}\left(y_{m}\right)}{\left(f^{\prime}\left(x_{m}\right)\right)^{2}-f^{\prime \prime}\left(y_{m}\right) f\left(y_{m}\right)}\right), \quad \beta>1 .
\end{gathered}
$$

BMM5: The ninth-order iterative method is based on the Halley's method [9] and the second step of the new ninth-order method is based on the Osada third order method [13], then the new ninth order iterative method is given as,

$$
\begin{gathered}
y_{m}=x_{m}-\frac{f\left(x_{m}\right)}{\frac{\beta+1}{2 \beta} f^{\prime}\left(x_{m}\right)-\frac{f\left(x_{m}\right) f^{\prime \prime}\left(x_{m}\right)}{2 f^{\prime}\left(x_{m}\right)}}, \quad \beta>1 . \\
x_{m+1}=y_{m}-\left(\frac{\beta(\beta-1)}{2} \frac{f\left(y_{m}\right)}{f^{\prime}\left(y_{m}\right)}-\frac{(\beta-1)^{2}}{2} \frac{f^{\prime}\left(y_{m}\right)}{f^{\prime \prime}\left(y_{m}\right)}\right) .
\end{gathered}
$$

BMM6: Another from of the ninth-order iterative method is based on the Halley's method [9] and the second step of the new ninth-order method is based on the Chune-Neta third-order method [14], then the new method of order nine is given as

$$
\begin{gathered}
y_{m}=x_{m}-\frac{f\left(x_{m}\right)}{\frac{\beta+1}{2 \beta} f^{\prime}\left(x_{m}\right)-\frac{f\left(x_{m}\right) f^{\prime \prime}\left(x_{m}\right)}{2 f^{\prime}\left(x_{m}\right)}}, \quad \beta>1 . \\
x_{m+1}=y_{m}-\left(2 \beta^{2}\left(f\left(y_{m}\right)\right)^{2} f^{\prime \prime}\left(y_{m}\right)\right)\left[\beta(3-\beta)^{2} f\left(y_{m}\right) f^{\prime}\left(y_{m}\right) f^{\prime \prime}\left(y_{m}\right)+(\beta-1)^{2}\left(f^{\prime}\left(y_{m}\right)\right)^{3}\right]^{-1} .
\end{gathered}
$$

BMM7: Finally, the ninth-order iterative method which is based on the third-order method [9], and the second step of the new ninth-order methods is based on the Euler-Chebyshev third-order method [15], then the new method of order nine is given as

$$
y_{m}=x_{m}-\frac{f\left(x_{m}\right)}{\frac{\beta+1}{2 \beta} f^{\prime}\left(x_{m}\right)-\frac{f\left(x_{m}\right) f^{\prime \prime}\left(x_{m}\right)}{2 f^{\prime}\left(x_{m}\right)}}, \quad \beta>1 .
$$




$$
x_{m+1}=y_{m}-\left(\frac{\beta(3-\beta)}{2} \frac{f\left(y_{m}\right)}{f^{\prime}\left(y_{m}\right)}+\frac{\beta^{2}}{2} \frac{\left(f\left(y_{m}\right)\right)^{2} f^{\prime \prime}\left(y_{m}\right)}{\left(f^{\prime}\left(y_{m}\right)\right)^{3}}\right) .
$$

We give the following convergence theorem for the above proposed methods as

Theorem Let $\alpha \in I$ be a real multiple zero of a sufficiently differentiable function $f: I \subset R \rightarrow R$ for an open interval $I$ with multiplicity $\beta$, which includes $x_{0}$ as an initial guess of $\alpha$, then the method.

(i) BMM1 defined by (5) has fourth-order convergence and the error equation for the scheme for is given as

$$
e_{m+1}=\left(\frac{c_{1}^{3}}{(\beta-1)^{3}}\right) e_{m}^{4}+O\left(e_{m}^{5}\right)
$$

(ii) BMM2 defined by (6) has sixth-order convergence, and the error equation for the scheme is given as

$$
e_{m+1}=\left(\frac{\left(\beta^{2}+2 \beta+1\right) c_{1}^{5}-\left(4 \beta^{2}+4 \beta\right) c_{1}^{3} c_{2}+4 \beta^{2} c_{1} c_{2}^{2}}{4 \beta^{4}(\beta-1)}\right) e_{m}^{6}+O\left(e_{m}^{7}\right) .
$$

(iii) BMM3, BMM4, BMM5, BMM6, and BMM7 defined by (11), (12), (13), (14) and (15), respectively have ninth-order convergence.

\section{Proof (i)}

Let $\alpha$ be a real multiple root of multiplicity $\beta$ of a sufficiently differentiable function $f(x)$ and $f(\alpha)=0$, we denote the errors given by each step as $e_{m}=x_{m}-\alpha, \tilde{e_{m}}=y_{m}-\alpha$. Using the Taylor series expansions of $f\left(x_{m}\right), f^{\prime}\left(x_{m}\right), f^{\prime \prime}\left(x_{m}\right), f\left(y_{m}\right), f^{\prime}\left(y_{m}\right)$ and $f^{\prime \prime}\left(y_{m}\right)$ about $\alpha$, we have:

$$
\begin{gathered}
f\left(x_{m}\right)=\left(\frac{f^{(\beta)}(\alpha)}{\beta !}\right) e_{m}{ }^{\beta}\left[1+c_{1} e_{m}+c_{2} e_{m}^{2}+c_{3} e_{m}^{3}+\ldots . .\right], \\
f^{\prime}\left(x_{m}\right)=\left(\frac{f^{(\beta)}(\alpha)}{(\beta-1) !}\right) e_{m}^{\beta-1}\left[1+\left(\frac{\beta+1}{\beta}\right) c_{1} e_{m}+\left(\frac{\beta+2}{\beta}\right) c_{2} e_{m}^{2}+\left(\frac{\beta+3}{\beta}\right) c_{3} e_{m}^{3}+\ldots . .\right], \\
f^{\prime \prime}\left(x_{m}\right)=\left(\frac{f^{(\beta)}(\alpha)}{(\beta-2) !}\right) e_{m}^{\beta-2}\left[1+\left(\frac{\beta+1}{\beta-1}\right) c_{1} e_{m}+\left(\frac{(\beta+1)(\beta+2)}{\beta(\beta-1)}\right) c_{2} e_{m}^{2}\right. \\
\left.+\left(\frac{(\beta+1)(\beta+2)(\beta+3)}{\beta(\beta-1)(\beta+1)}\right) c_{3} e_{m}^{3}+\ldots . .\right], \\
f\left(y_{m}\right)=\left(\frac{f^{(\beta)}(\alpha)}{\beta !}\right) \tilde{e}_{m}^{\beta}\left[1+c_{1} \tilde{e}_{m}+c_{2} \tilde{e}_{m}^{2}+c_{3} \tilde{e}_{m}^{3}+\ldots . .\right], \\
f^{\prime}\left(y_{m}\right)=\left(\frac{f^{(\beta)}(\alpha)}{(\beta-1) !}\right) \tilde{e}_{m}^{\beta-1}\left[1+\left(\frac{\beta+1}{\beta}\right) c_{1} \tilde{e}_{m}+\left(\frac{\beta+2}{\beta}\right) c_{2} \tilde{e}_{m}^{2}+\left(\frac{\beta+3}{\beta}\right) c_{3} \tilde{e}_{m}^{3}+\ldots . .\right], \\
f^{\prime \prime}\left(y_{m}\right)=\left(\frac{f^{(\beta)}(\alpha)}{(\beta-2) !}\right) \tilde{e}_{m}^{\beta-2}\left[1+\left(\frac{\beta+1}{\beta-1}\right) c_{1} \tilde{e}_{m}\right. \\
\left.+\left(\frac{(\beta+1)(\beta+2)}{\beta(\beta-1)}\right) c_{2} \tilde{e}_{m}^{2}+\left(\frac{(\beta+1)(\beta+2)(\beta+3)}{\beta(\beta-1)(\beta+1)}\right) c_{3} \tilde{e}_{m}^{3}+\ldots\right],
\end{gathered}
$$

where $m \in N$ and $c_{i}=\frac{\beta ! f^{(\beta+i)}(\alpha)}{(\beta+i) ! f^{(\beta)}(\alpha)}, \quad i=1,2, \ldots$. Furthermore, we have

$$
\begin{gathered}
\frac{f\left(x_{m}\right)}{f^{\prime}\left(x_{m}\right)}=\frac{1}{\beta} e_{m}-\frac{c_{1}}{\beta^{2}} e_{m}^{2}+\frac{(\beta+1) c_{1}^{2}-2 \beta c_{2}}{\beta^{3}} e_{m}^{3}-\frac{\left(\beta^{2} c_{1}^{3}-3 \beta^{2} c_{1} c_{2}+2 \beta c_{1}^{3}+3 \beta^{2} c_{3}-4 \beta c_{1} c_{2}+c_{1}^{3}\right)}{\beta^{4}} e_{m}^{4}+\ldots \\
\frac{f^{\prime}\left(x_{m}\right)}{f^{\prime \prime}\left(x_{m}\right)}=\frac{1}{\beta-1} e_{m}-\frac{(\beta+1) c_{1}}{\beta(\beta-1)^{2}} e_{m}^{2}+\frac{\left(\beta^{2}+2 \beta+1\right) c_{1}^{2}-\left(2 \beta^{2}+2 \beta-4\right) c_{2}}{\beta(\beta-1)^{3}} e_{m}^{3}
\end{gathered}
$$




$$
\begin{aligned}
& -\left(\frac{1}{\beta^{2}(\beta-1)^{4}}\right)\left(\beta^{4} c_{1}^{3}-3 \beta^{4} c_{1} c_{2}+3 \beta^{3} c_{1}^{3}+3 \beta^{4} c_{3}-7 \beta^{3} c_{1} c_{2}\right. \\
& \left.+3 \beta^{2} c_{1}^{3}+3 \beta^{3} c_{3}+\beta^{2} c_{1} c_{2}+\beta c_{1}^{3}-15 \beta^{2} c_{3}+7 \beta c_{1} c_{2}+9 \beta c_{3}+2 c_{1} c_{2}\right) e_{m}^{4}+\ldots
\end{aligned}
$$

Moreover by (5), we have

$$
\begin{aligned}
y_{m} & =x_{m}-\frac{1}{2}\left(\beta \frac{f\left(x_{m}\right)}{f^{\prime}\left(x_{m}\right)}+(\beta-1) \frac{f^{\prime}\left(x_{m}\right)}{f^{\prime \prime}\left(x_{m}\right)}\right) \\
& =\alpha+\left(\frac{c_{1}}{\beta-1}\right) e_{m}^{2}-\left(\frac{\left(2 \beta^{3}+\beta^{2}+1\right) c_{1}^{2}-\left(4 \beta^{3}-2 \beta^{2}-2 \beta\right) c_{2}}{2 \beta^{2}(\beta-1)^{2}}\right) e_{m}^{3} \\
& +\left(\frac{1}{2 \beta^{3}(\beta-1)^{3}}\right)\left(\left(2 \beta^{5}+2 \beta^{4}+\beta^{3}+3 \beta^{2}+\beta-1\right) c_{1}^{3}\right. \\
& \left.-\left(6 \beta^{5}+2 \beta^{4}-4 \beta^{3}+2 \beta^{2}-6 \beta\right) c_{1} c_{2}+\left(6 \beta^{5}-6 \beta^{4}-6 \beta^{3}+6 \beta^{2}\right) c_{3}\right) e_{m}^{4}+\ldots . .
\end{aligned}
$$

The expansions of $f\left(y_{m}\right), f^{\prime}\left(y_{m}\right)$ and $f^{\prime \prime}\left(y_{m}\right)$ about $\alpha$ and simplifying, yield

$$
\begin{aligned}
f\left(y_{m}\right)=\frac{f^{(\beta)}(\alpha)}{\beta !}\left(\frac{c_{1}}{(\beta-1)}\right)^{\beta}\left(e_{m}^{2}\right)^{\beta}\left[1+\frac{c_{1}^{2}}{m-1} e_{m}^{2}-\frac{1}{2} \frac{c_{1}^{3}\left(2 \beta^{3}+\beta^{2}+1\right)}{\beta^{2}(\beta-1)^{2}} e_{m}^{3}+\ldots\right] & \\
f^{\prime}\left(y_{m}\right) & =\frac{f^{(\beta)}(\alpha)}{(\beta-1) !}\left(\frac{c_{1}}{(\beta-1)}\right)^{(\beta-1)}\left(e_{m}^{2}\right)^{(\beta-1)}\left[\beta+\frac{(\beta+1) c_{1}^{2}}{m-1} e_{m}^{2}\right. \\
& \left.-\frac{1}{2} \frac{c_{1}^{3}\left(2 \beta^{4}+3 \beta^{3}+\beta^{2}+\beta+1\right)}{\beta^{2}(\beta-1)^{2}} e_{m}^{3}+\ldots\right] \\
f^{\prime \prime}\left(y_{m}\right) & =\frac{f^{(\beta)}(\alpha)}{(\beta-2) !}\left(\frac{c_{1}}{(\beta-1)}\right)^{(\beta-2)}\left(e_{m}^{2}\right)^{(\beta-2)}\left[\beta^{2}-\beta+\frac{(\beta+1) \beta c_{1}^{2}}{m-1} e_{m}^{2}\right. \\
& \left.-\frac{1}{2} \frac{c_{1}^{3}\left(2 \beta^{4}+3 \beta^{3}+\beta^{2}+\beta+1\right)}{\beta(\beta-1)^{2}} e_{m}^{3}+\ldots\right]
\end{aligned}
$$

So, we can get

$$
\begin{aligned}
\frac{f\left(y_{m}\right)}{f^{\prime}\left(y_{m}\right)} & =\left(\frac{c_{1}}{\beta(\beta-1)}\right) e_{m}^{2}-\left(\frac{1}{2 \beta^{3}(\beta-1)^{2}}\right)\left(\left(2 \beta^{3}+\beta^{2}+1\right) c_{1}^{2}\right. \\
& \left.-\left(4 \beta^{3}-2 \beta^{2}-2 \beta\right) c_{2}\right) e_{m}^{3}+\ldots, \\
\frac{f^{\prime}\left(y_{m}\right)}{f^{\prime \prime}\left(y_{m}\right)} & =\frac{c_{1}}{(\beta-1)^{2}} e_{m}^{2}-\left(\frac{1}{2 \beta^{2}(\beta-1)^{3}}\right)\left(\left(2 \beta^{3}+\beta^{2}+1\right) c_{1}^{2}\right. \\
& \left.-\left(4 \beta^{3}-2 \beta^{2}-2 \beta\right) c_{2}\right) e_{m}^{3}+\ldots,
\end{aligned}
$$

Submitting (18), (19) and (20) into the second part of (5), we have

$$
x_{m+1}=\alpha+\left(\frac{c_{1}^{3}}{(\beta-1)^{3}}\right) e_{m}^{4}+O\left(e_{m}^{5}\right),
$$


simplifying (21), we obtain the asymptotic error constant

$$
e_{m+1}=\left(\frac{c_{1}^{3}}{(\beta-1)^{3}}\right) e_{m}^{4}+O\left(e_{m}^{5}\right)
$$

The expression (22) establishes the asymptotic error constant for the fourth order of convergence for the method BMM1 defined by (5) .

Proof (ii)

Using appropriate expressions in the proof of (i) and substituting them into (6), we have

$$
\begin{aligned}
y_{m} & =\alpha+\left(\frac{(\beta+1) c_{1}^{2}-2 \beta c_{2}}{2 \beta^{2}}\right) e_{m}^{3}-\left(\frac{1}{2 \beta^{3}}\right)\left(\left(2 \beta^{2}+3 \beta+1\right) c_{1}^{3}\right. \\
& \left.-\left(6 \beta^{2}+6 \beta\right) c_{1} c_{2}+6 \beta^{2} c_{3}\right) e_{m}^{4}+\ldots .
\end{aligned}
$$

The expansions of $f\left(y_{m}\right), f^{\prime}\left(y_{m}\right)$ and $f^{\prime \prime}\left(y_{m}\right)$ about $\alpha$ and simplifying, yield

$$
\begin{aligned}
f\left(y_{m}\right) & =\frac{f^{(\beta)}(\alpha)}{\beta !}\left(\frac{c_{1}\left(\beta c_{1}^{2}-2 \beta c_{2}+c_{1}^{2}\right)}{2 \beta^{2}}\right)^{\beta}\left(e_{m}^{3}\right)^{\beta}\left[1+\frac{c_{1}\left(\beta c_{1}^{2}-2 \beta c_{2}+c_{1}^{2}\right)}{2 \beta^{2}} e_{m}^{3}\right. \\
& \left.-\frac{c_{1}\left(2 \beta^{2} c_{1}^{3}-6 \beta^{2} c_{1} c_{2}+3 \beta c_{1}^{3}+6 \beta^{2} c_{3}-6 \beta c_{1} c_{2}+c_{1}^{3}\right)}{2 \beta^{3}} e_{m}^{4}+\ldots\right] \\
f^{\prime}\left(y_{m}\right)= & \frac{f^{(\beta)}(\alpha)}{(\beta-1) !}\left(\frac{c_{1}\left(\beta c_{1}^{2}-2 \beta c_{2}+c_{1}^{2}\right)}{2 \beta^{2}}\right)^{\beta-1}\left(e_{m}^{3}\right)^{\beta-1}\left[\beta+\frac{c_{1}\left(\beta^{2} c_{1}^{2}-2 \beta^{2} c_{2}+2 \beta c_{1}^{2}-2 \beta c_{2}+c_{1}^{2}\right)}{2 \beta^{2}} e_{m}^{3}\right. \\
- & \left.\frac{c_{1}\left(2 \beta^{3} c_{1}^{3}-6 \beta^{3} c_{1} c_{2}+5 \beta^{2} c_{1}^{3}+6 \beta^{3} c_{3}-12 \beta^{2} c_{1} c_{2}+4 \beta c_{1}^{3}+6 \beta^{2} c_{3}-6 \beta c_{1} c_{2}+c_{1}^{3}\right)}{2 \beta^{3}} e_{m}^{4}+\ldots\right] \\
f^{\prime \prime}\left(y_{m}\right)= & \frac{f^{(\beta)}(\alpha)}{(\beta-2) !}\left(\frac{c_{1}\left(\beta c_{1}^{2}-2 \beta c_{2}+c_{1}^{2}\right)}{2 \beta^{2}}\right)^{\beta-2}\left(e_{m}^{3}\right)^{\beta-2}[ \\
& \beta^{2}-\beta+\frac{c_{1}\left(\beta^{2} c_{1}^{2}-2 \beta^{2} c_{2}+2 \beta c_{1}^{2}-2 \beta c_{2}+c_{1}^{2}\right)}{2 \beta} e_{m}^{3} \\
- & \left.\frac{c_{1}\left(2 \beta^{3} c_{1}^{3}-6 \beta^{3} c_{1} c_{2}+5 \beta^{2} c_{1}^{3}+6 \beta^{3} c_{3}-12 \beta^{2} c_{1} c_{2}+4 \beta c_{1}^{3}+6 \beta^{2} c_{3}-6 \beta c_{1} c_{2}+c_{1}^{3}\right)}{2 \beta^{2}} e_{m}^{4}+\ldots\right]
\end{aligned}
$$

So, we can get

$$
\begin{aligned}
\frac{f\left(y_{m}\right)}{f^{\prime}\left(y_{m}\right)} & =\left(\frac{(\beta+1) c_{1}^{2}-2 \beta c_{2}}{2 m^{3}}\right) e_{m}^{3}-\left(\frac{1}{2 \beta^{4}}\right)\left(\left(2 \beta^{2}+3 \beta+1\right) c_{1}^{3}\right. \\
& \left.-\left(6 \beta^{2}+6 \beta\right) c_{1} c_{2}+6 \beta^{2} c_{3}\right) e_{m}^{4}+\ldots ., \\
\frac{f^{\prime}\left(y_{m}\right)}{f^{\prime \prime}\left(y_{m}\right)}= & \left(\frac{(\beta+1) c_{1}^{2}-2 \beta c_{2}}{2 \beta^{2}(\beta-1)^{2}}\right) e_{m}^{3}-\left(\frac{1}{2 \beta^{3}(\beta-1)}\right)\left(\left(2 \beta^{2}+3 \beta+1\right) c_{1}^{3}\right. \\
& \left.-\left(6 \beta^{2}+6 \beta\right) c_{1} c_{2}+6 \beta^{2} c_{3}\right) e_{m}^{4}+\ldots .,
\end{aligned}
$$

Submitting (23), (24) and (25) into the second part of (6), we have

$$
x_{m+1}=\alpha+\left(\frac{\left(\beta^{2}+2 \beta+1\right) c_{1}^{5}-\left(4 \beta^{2}+4 \beta\right) c_{1}^{3} c_{2}+4 \beta^{2} c_{1} c_{2}^{2}}{4 \beta^{4}(\beta-1)}\right) e_{m}^{6}+O\left(e_{m}^{7}\right),
$$


simplifying (26), we obtain the asymptotic error constant

$$
e_{m+1}=\left(\frac{\left(\beta^{2}+2 \beta+1\right) c_{1}^{5}-\left(4 \beta^{2}+4 \beta\right) c_{1}^{3} c_{2}+4 \beta^{2} c_{1} c_{2}^{2}}{4 \beta^{4}(\beta-1)}\right) e_{m}^{6}+O\left(e_{m}^{7}\right),
$$

this establishes the asymptotic error constant for the sixth order method of convergence for the new Halley's method [9], defined by (6).

\section{Proof (iii) (BMM3)}

Using appropriate expressions in the proofs of (i) and (ii) and substituting them into (11), we obtain the asymptotic error constant

$$
\begin{aligned}
e_{m+1} & =\frac{1}{16 \beta^{8}(\beta-1)^{4}}\left(\left(\beta^{8}-8 \beta^{7}+12 \beta^{6}+40 \beta^{5}-74 \beta^{4}-120 \beta^{3}+180 \beta^{2}+216 \beta+81\right) c_{1}^{8}\right. \\
& -\left(8 \beta^{8}-56 \beta^{7}+72 \beta^{6}+200 \beta^{5}-296 \beta^{4}-360 \beta^{3}+216 \beta^{2}+216 \beta\right) c_{1}^{6} c_{2} \\
& +\left(24 \beta^{8}-144 \beta^{7}+168 \beta^{6}+288 \beta^{5}-408 \beta^{4}-144 \beta^{3}+216 \beta^{2}\right) c_{1}^{4} c_{2}^{2} \\
& -\left(32 \beta^{8}-160 \beta^{7}+192 \beta^{6}+64 \beta^{5}-224 \beta^{4}+96 \beta^{3}\right) c_{1}^{2} c_{2}^{3} \\
& \left.+\left(16 \beta^{8}-64 \beta^{7}+96 \beta^{6}+74 \beta^{5}+16 \beta^{4}\right) c_{2}^{4}\right) e_{m}^{9}+O\left(e_{m}^{10}\right),
\end{aligned}
$$

which shows that BMM3 defined by (11) has ninth-order convergence.

In a similar way and using appropriate expressions in the proof of (i) and (ii), we observe that the methods BMM4, BMM5, BMM6 and BMM7 have also ninth order convergence and the error equation of these methods is as follows:

$$
\begin{aligned}
e_{m+1} & =\frac{1}{16 \beta^{8}(\beta-1)}\left(\left(\beta^{5}+\beta^{4}-6 \beta^{3}-14 \beta^{2}-11 \beta-3\right) c_{1}^{8}\right. \\
& -\left(8 \beta^{5}+4 \beta^{4}-36 \beta^{3}-52 \beta^{2}-20 \beta\right) c_{1}^{6} c_{2}+\left(24 \beta^{5}-72 \beta^{3}-48 \beta^{2}\right) c_{1}^{4} c_{2}^{2} \\
& \left.-\left(32 \beta^{5}-16 \beta^{4}-48 \beta^{3}\right) c_{1}^{2} c_{2}^{3}+\left(16 \beta^{5}-16 \beta^{4}\right) c_{2}^{4}\right) e_{m}^{9}+O\left(e_{m}^{10}\right) . \quad \text { (BMM4) } \\
e_{m+1} & =\frac{1}{16 \beta^{8}(\beta-1)}\left(\left(\beta^{5}+5 \beta^{4}+10 \beta^{3}+10 \beta^{2}+5 \beta+1\right) c_{1}^{8}\right. \\
& -\left(8 \beta^{5}+28 \beta^{4}+36 \beta^{3}+20 \beta^{2}+4 \beta\right) c_{1}^{6} c_{2}+\left(24 \beta^{5}+48 \beta^{4}+24 \beta^{3}\right) c_{1}^{4} c_{2}^{2} \\
& \left.-\left(32 \beta^{5}+16 \beta^{4}-16 \beta^{3}\right) c_{1}^{2} c_{2}^{3}+\left(16 \beta^{5}-16 \beta^{4}\right) c_{2}^{4}\right) e_{m}^{9}+O\left(e_{m}^{10}\right) . \quad \text { (BMM5) } \\
& =\frac{1}{16 \beta^{8}(\beta-1)}\left(\left(\beta^{5}+3 \beta^{4}+6 \beta^{3}+10 \beta^{2}+9 \beta+3\right) c_{1}^{8}\right. \\
- & \left(8 \beta^{5}+16 \beta^{4}+24 \beta^{3}+32 \beta^{2}+16 \beta\right) c_{1}^{6} c_{2}+\left(24 \beta^{5}+24 \beta^{4}+24 \beta^{3}+24 \beta^{2}\right) c_{1}^{4} c_{2}^{2} \\
e_{m+1} & \left.32 \beta^{5} c_{1}^{2} c_{2}^{3}+\left(16 \beta^{5}-16 \beta^{4}\right) c_{2}^{4}\right) e_{m}^{9}+O\left(e_{m}^{10}\right) . \\
& \quad \text { (BMM6) } \\
e_{m+1} & =\frac{1}{16 \beta^{8}}\left(\left(\beta^{4}+6 \beta^{3}+12 \beta^{2}+10 \beta+3\right) c_{1}^{8}\right. \\
& -\left(8 \beta^{4}+36 \beta^{3}+48 \beta^{2}+20 \beta\right) c_{1}^{6} c_{2}+\left(24 \beta^{4}+72 \beta^{3}+48 \beta^{2}\right) c_{1}^{4} c_{2}^{2} \\
& \left.-\left(32 \beta^{4}+48 \beta^{3}\right) c_{1}^{2} c_{2}^{3}+16 \beta^{4} c_{2}^{4}\right) e_{m}^{9}+O\left(e_{m}^{10}\right) .
\end{aligned}
$$

Also, we are using the variational iteration technique to suggest some one-step iterative algorithms for finding the multiple roots of the nonlinear equations. In this study, we consider $\phi\left(x_{n}\right)$ is as an iteration auxiliary function of order $p \geq 1$, with $\beta$ being the multiplicity of the zero of $f(x)$ and $\lambda$ unknown Lagrange's multiplier (see $[2,9,13,14,16-22]$ ), such that.

$$
x=\phi(x)+\lambda[f(x) g(x)]^{\frac{p}{\beta}} .
$$


This fixed point function enables to suggest the following scheme for finding the approximate solution of $f(x)=0$ by

$$
x_{m+1}=\phi\left(x_{m}\right)+\lambda\left[f\left(x_{m}\right) g\left(x_{m}\right)\right]^{\frac{p}{\beta}},
$$

Using the optimality condition, we have

$$
\lambda=-\frac{\beta \phi^{\prime}\left(x_{m}\right)}{p\left[f\left(x_{m}\right) g\left(x_{m}\right)\right]^{\frac{p}{\beta}-1}\left[f^{\prime}\left(x_{m}\right) g\left(x_{m}\right)+f\left(x_{m}\right) g^{\prime}\left(x_{m}\right)\right]},
$$

From (34) and (35), we have

$$
x_{m+1}=\phi\left(x_{m}\right)-\frac{\beta \phi^{\prime}\left(x_{m}\right) f\left(x_{m}\right) g\left(x_{m}\right)}{p\left[f^{\prime}\left(x_{m}\right) g\left(x_{m}\right)+f\left(x_{m}\right) g^{\prime}\left(x_{m}\right)\right]} .
$$

This is a recurrence relation which generates iterative algorithms of order $p=1$ for solving the nonlinear equation $f(x)=0$. We use (36) to suggest several iterative algorithms of higher order for finding multiple roots of the nonlinear equation and this is the main motivation. We now discuss some special cases.

\section{Case 1}

In this case, we will modify (36) for obtaining iterative algorithms of second and third order for solving nonlinear equations. Let

$$
\begin{gathered}
\phi\left(x_{m}\right)=x_{m}-\left[\frac{2 \beta}{2+\beta} \cdot \frac{f\left(x_{m}\right)}{f^{\prime}\left(x_{m}\right)},\right] \\
\phi^{\prime}\left(x_{m}\right)=\frac{(2-\beta)\left(f^{\prime}\left(x_{m}\right)\right)^{2}+2 \beta f\left(x_{m}\right) f^{\prime \prime}\left(x_{m}\right)}{(\beta+2)\left(f^{\prime}\left(x_{m}\right)\right)^{2}},
\end{gathered}
$$

where $p=1$ is the order of convergence for $\phi\left(x_{m}\right)$. Then relation (36) becomes,

$$
x_{m+1}=x_{m}-\frac{2 \beta\left(f\left(x_{m}\right)\right)^{2} f^{\prime}\left(x_{m}\right) g^{\prime}\left(x_{m}\right)+\left[2 \beta^{2}\left(f\left(x_{m}\right)\right)^{2} f^{\prime \prime}\left(x_{m}\right)+\left(4 \beta-\beta^{2}\right) f\left(x_{m}\right)\left(f^{\prime}\left(x_{m}\right)\right)^{2}\right] g\left(x_{m}\right)}{(\beta+2)\left[\left(f^{\prime}\left(x_{m}\right)\right)^{3} g\left(x_{m}\right)+f\left(x_{m}\right)\left(f^{\prime}\left(x_{m}\right)\right)^{2} g^{\prime}\left(x_{m}\right)\right]} .
$$

This is the iteration relation which generates at least second order convergent algorithms for finding multiple roots of the nonlinear equation. Now, we consider some special values of $g\left(x_{m}\right)$ to suggest some iterative algorithms for solving nonlinear equation $f(x)=0$.

i) Let $g\left(x_{m}\right)=e^{-\xi x_{m}}, \quad g^{\prime}\left(x_{m}\right)=-\xi e^{-\xi x_{m}}$, then from (37), we obtain the following iterative algorithm for finding the multiple roots of the nonlinear equation

BMM8 For a given $x_{0}$, we find the approximate solution $x_{m+1}$ by the iterative algorithm

$$
x_{m+1}=x_{m}-\frac{2 \beta^{2}\left(f\left(x_{m}\right)\right)^{2} f^{\prime \prime}\left(x_{m}\right)+\left(4 \beta-\beta^{2}\right) f\left(x_{m}\right)\left(f^{\prime}\left(x_{m}\right)\right)^{2}-2 \beta \xi\left(f\left(x_{m}\right)\right)^{2} f^{\prime}\left(x_{m}\right)}{(\beta+2)\left[\left(f^{\prime}\left(x_{m}\right)\right)^{3}-\xi f\left(x_{m}\right)\left(f^{\prime}\left(x_{m}\right)\right)^{2}\right]} .
$$

If $\xi=0, \mathrm{BMM} 8$ reduces to the following second-order convergent iterative algorithm as the form

$$
x_{m+1}=x_{m}-\frac{2 \beta^{2}\left(f\left(x_{m}\right)\right)^{2} f^{\prime \prime}\left(x_{m}\right)+\left(4 \beta-\beta^{2}\right) f\left(x_{m}\right)\left(f^{\prime}\left(x_{m}\right)\right)^{2}}{(\beta+2)\left(f^{\prime}\left(x_{m}\right)\right)^{3}}
$$

The error equation for the algorithm is given by

$$
e_{m+1}=\frac{(\beta-2) c_{1}}{(\beta+2) \beta} e_{m}^{2}+O\left(e_{m}^{3}\right)
$$

If $\xi=\frac{(\beta-2) c_{1}}{\beta}$, BMM8 reduces to the following third-order convergent iterative algorithm:

$$
x_{m+1}=x_{m}-\frac{2 \beta^{2}\left(f\left(x_{m}\right)\right)^{2} f^{\prime \prime}\left(x_{m}\right)+\left(4 \beta-\beta^{2}\right) f\left(x_{m}\right)\left(f^{\prime}\left(x_{m}\right)\right)^{2}-2 \beta\left(\frac{(\beta-2) c_{1}}{\beta}\right)\left(f\left(x_{m}\right)\right)^{2} f^{\prime}\left(x_{m}\right)}{(\beta+2)\left[\left(f^{\prime}\left(x_{m}\right)\right)^{3}-\left(\left(\frac{(\beta-2) c_{1}}{\beta}\right) f\left(x_{m}\right)\left(f^{\prime}\left(x_{m}\right)\right)^{2}\right)\right]}
$$


The error equation for this algorithm is

$$
e_{m+1}=-\frac{\left(\beta^{3}-4 \beta^{2}-4 \beta-4\right) c_{1}^{2}-\left(2 \beta^{3}-8 \beta^{2}\right) c_{2}}{(\beta+2) \beta^{3}} e_{m}^{3}+\ldots
$$

ii) Let $g\left(x_{m}\right)=e^{\frac{-\xi f\left(x_{m}\right)}{f^{\prime}\left(x_{m}\right)}}, \quad g^{\prime}\left(x_{m}\right)=-\xi \frac{\left(f^{\prime}\left(x_{m}\right)\right)^{2}-f\left(x_{m}\right) f^{\prime \prime}\left(x_{m}\right)}{\left(f^{\prime}\left(x_{m}\right)\right)^{2}} e^{\frac{-\xi f\left(x_{m}\right)}{f^{\prime}\left(x_{m}\right)}}$. Then relation (37) becomes

BMM9

$$
x_{m+1}=x_{m}-\frac{2 \beta^{2}\left(f\left(x_{m}\right)\right)^{2} f^{\prime \prime}\left(x_{m}\right)+\left(4 \beta-\beta^{2}\right) f\left(x_{m}\right)\left(f^{\prime}\left(x_{m}\right)\right)^{2}-\xi\left(\frac{2 \beta f\left(x_{m}\right)\left[f\left(x_{m}\right)\left(f^{\prime}\left(x_{m}\right)\right)^{2}-\left(f\left(x_{m}\right)\right)^{2} f^{\prime \prime}\left(x_{m}\right)\right]}{f^{\prime}\left(x_{m}\right)}\right)}{(\beta+2)\left[\left(f^{\prime}\left(x_{m}\right)\right)^{3}-\xi\left(f\left(x_{m}\right)\left(f^{\prime}\left(x_{m}\right)\right)^{2}-\left(f\left(x_{m}\right)\right)^{2} f^{\prime \prime}\left(x_{m}\right)\right)\right]} .
$$

If $\xi=0$, BMM9 reduces to the following second-order convergent iterative algorithm

$$
x_{m+1}=x_{m}-\frac{2 \beta^{2}\left(f\left(x_{m}\right)\right)^{2} f^{\prime \prime}\left(x_{m}\right)+\left(4 \beta-\beta^{2}\right) f\left(x_{m}\right)\left(f^{\prime}\left(x_{m}\right)\right)^{2}}{(\beta+2)\left(f^{\prime}\left(x_{m}\right)\right)^{3}} .
$$

The error equation for this algorithm is

$$
e_{m+1}=\frac{(\beta-2) c_{1}}{(\beta+2) \beta} e_{m}^{2}+O\left(e_{m}^{3}\right)
$$

If $\xi=\frac{(\beta-2) c_{1} f\left(x_{m}\right)\left(f^{\prime}\left(x_{m}\right)\right)^{2}}{\beta\left[f\left(x_{m}\right)\left(f^{\prime}\left(x_{m}\right)\right)^{2}-\left(f\left(x_{m}\right)\right)^{2} f^{\prime \prime}\left(x_{m}\right)\right]}$, then BMM9 reduces to the following third-order convergent iterative algorithm for finding simple root of nonlinear equations.

$$
x_{m+1}=x_{m}-\frac{2 \beta^{2}\left(f\left(x_{m}\right)\right)^{2} f^{\prime \prime}\left(x_{m}\right)+\left(4 \beta-\beta^{2}\right) f\left(x_{m}\right)\left(f^{\prime}\left(x_{m}\right)\right)^{2}-2 \beta\left(\frac{(\beta-2) c_{1}}{\beta}\right)\left(f\left(x_{m}\right)\right)^{2} f^{\prime}\left(x_{m}\right)}{(\beta+2)\left[\left(f^{\prime}\left(x_{m}\right)\right)^{3}-\left(\left(\frac{(\beta-2) c_{1}}{\beta}\right) f\left(x_{m}\right)\left(f^{\prime}\left(x_{m}\right)\right)^{2}\right)\right]} .
$$

The error equation for the algorithm is given by

$$
e_{m+1}=-\frac{\left(\beta^{3}-4 \beta^{2}-4 \beta-4\right) c_{1}^{2}-\left(2 \beta^{3}-8 \beta^{2}\right) c_{2}}{(\beta+2) \beta^{3}} e_{m}^{3}+\ldots
$$

\section{Case 2}

In this case, we will modify (36) for obtaining iterative algorithms of second and third order for solving nonlinear equations. Let

$$
\begin{gathered}
\phi\left(x_{m}\right)=x_{m}-\sqrt{\beta} \frac{f\left(x_{m}\right)}{f^{\prime}\left(x_{m}\right)}, \\
\phi^{\prime}\left(x_{m}\right)=1-\sqrt{\beta}\left(1-\frac{f\left(x_{m}\right) f^{\prime \prime}\left(x_{m}\right)}{\left(f^{\prime}\left(x_{m}\right)\right)^{2}}\right),
\end{gathered}
$$

where $p=1$ is the order of convergence for $\phi\left(x_{m}\right)$, then relation (36) becomes

$$
x_{m+1}=x_{m}-\frac{g\left(x_{m}\right)\left[\beta \sqrt{\beta}\left(f\left(x_{m}\right)\right)^{2} f^{\prime \prime}\left(x_{m}\right)+(\beta+\sqrt{\beta}-\beta \sqrt{\beta}) f\left(x_{m}\right)\left(f^{\prime}\left(x_{m}\right)\right)^{2}\right]+\sqrt{\beta}\left(f\left(x_{m}\right)\right)^{2} f^{\prime}\left(x_{m}\right) g^{\prime}\left(x_{m}\right)}{g\left(x_{m}\right)\left(f^{\prime}\left(x_{m}\right)\right)^{3}+g^{\prime}\left(x_{m}\right) f\left(x_{m}\right)\left(f^{\prime}\left(x_{m}\right)\right)^{2}} .
$$

i) Let $g\left(x_{m}\right)=e^{-\xi x_{m}} \quad, \quad g^{\prime}\left(x_{m}\right)=-\xi e^{-\xi x_{m}}$, then, from (40), we obtain the following iterative algorithm:

BMM10

$$
x_{m+1}=x_{m}-\frac{\beta \sqrt{\beta}\left(f\left(x_{m}\right)\right)^{2} f^{\prime \prime}\left(x_{m}\right)+(\beta+\sqrt{\beta}-\beta \sqrt{\beta}) f\left(x_{m}\right) f^{\prime}\left(x_{m}\right)^{2}-\xi \sqrt{\beta}\left(f\left(x_{m}\right)\right)^{2} f^{\prime}\left(x_{m}\right)}{\left(f^{\prime}\left(x_{m}\right)\right)^{3}-\xi f\left(x_{m}\right)\left(f^{\prime}\left(x_{m}\right)\right)^{2}} .
$$

If $\xi=0$, BMM10 reduces to the following second-order convergent iterative algorithm as the form.

$$
x_{m+1}=x_{m}-\frac{\beta \sqrt{\beta}\left(f\left(x_{m}\right)\right)^{2} f^{\prime \prime}\left(x_{m}\right)+(\beta+\sqrt{\beta}-\beta \sqrt{\beta}) f\left(x_{m}\right)\left(f^{\prime}\left(x_{m}\right)\right)^{2}}{\left(f^{\prime}\left(x_{m}\right)\right)^{3}} .
$$


The error equation for this algorithm is

$$
e_{m+1}=\frac{(\sqrt{\beta}-2) c_{1}}{\beta \sqrt{\beta}} e_{m}^{2}+\cdots
$$

If $\xi=\frac{-(\sqrt{\beta}-2) c_{1}}{1-\sqrt{\beta}}$, then BMM10 reduces to the following third-order convergent iterative algorithm:

$$
x_{m+1}=x_{m}-\frac{\beta \sqrt{\beta}\left(f\left(x_{m}\right)\right)^{2} f^{\prime \prime}\left(x_{m}\right)+(\beta+\sqrt{\beta}-\beta \sqrt{\beta}) f\left(x_{m}\right)\left(f^{\prime}\left(x_{m}\right)\right)^{2}+\left(\frac{(\sqrt{\beta}-2) c_{1}}{1-\sqrt{\beta}}\right) \sqrt{\beta}\left(f\left(x_{m}\right)\right)^{2} f^{\prime}\left(x_{m}\right)}{\left(f^{\prime}\left(x_{m}\right)\right)^{3}+\left(\frac{(\sqrt{\beta}-2) c_{1}}{1-\sqrt{\beta}}\right) f\left(x_{m}\right)\left(f^{\prime}\left(x_{m}\right)\right)^{2}} .
$$

The error equation for this algorithm is given by

$$
e_{m+1}=\frac{\left[\beta(5 \beta+5)-\sqrt{\beta}\left(\beta^{2}+7 \beta+3\right)+1\right] c_{1}^{2}+\left[\sqrt{\beta}\left(14 \beta+2 \beta^{2}\right)-\beta(10 \beta+6)\right] c_{2}}{\beta \sqrt{\beta}(\sqrt{\beta}-1)^{2}} e_{m}^{3}+\cdots
$$

ii) Let $g\left(x_{m}\right)=e^{-\xi \frac{f\left(x_{m}\right)}{f^{\prime}\left(x_{m}\right)}}, g^{\prime}\left(x_{m}\right)=-\xi e^{-\xi \frac{f\left(x_{m}\right)}{f^{\prime}\left(x_{m}\right)}} \frac{\left(f^{\prime}\left(x_{m}\right)\right)^{2}-f\left(x_{m}\right) f^{\prime \prime}\left(x_{m}\right)}{\left(f^{\prime}\left(x_{m}\right)\right)^{2}}$.

Then from (40), we obtain the following iterative algorithm for finding the multiple roots of the nonlinear equation:

BMM11

$$
x_{m+1}=x_{m}-\frac{\beta \sqrt{\beta}\left(f\left(x_{m}\right)\right)^{2} f^{\prime \prime}\left(x_{m}\right)+(\beta+\sqrt{\beta}-\beta \sqrt{\beta}) f\left(x_{m}\right)\left(f^{\prime}\left(x_{m}\right)\right)^{2}-\sqrt{\beta} \xi f\left(x_{m}\right) h(x)}{\left(f^{\prime}\left(x_{m}\right)\right)^{3}-\xi\left(f\left(x_{m}\right)\left(f^{\prime}\left(x_{m}\right)\right)^{2}-\left(f\left(x_{m}\right)\right)^{2} f^{\prime \prime}\left(x_{m}\right)\right)},
$$

where $h(x)=\left(\frac{f\left(x_{m}\right)\left(f^{\prime}\left(x_{m}\right)\right)^{2}-\left(f\left(x_{m}\right)\right)^{2} f^{\prime \prime}\left(x_{m}\right)}{f^{\prime}\left(x_{m}\right)}\right)$. If $\xi=0$, then BMM11 reduces to the following second-order convergent iterative algorithm

$$
x_{m+1}=x_{m}-\frac{\beta \sqrt{\beta}\left(f\left(x_{m}\right)\right)^{2} f^{\prime \prime}\left(x_{m}\right)+(\beta+\sqrt{\beta}-\beta \sqrt{\beta}) f\left(x_{m}\right)\left(f^{\prime}\left(x_{m}\right)\right)^{2}}{\left(f^{\prime}\left(x_{m}\right)\right)^{3}} .
$$

The error equation for the method is given by

$$
e_{m+1}=\frac{(\sqrt{\beta}-2) c_{1}}{\beta \sqrt{\beta}} e_{m}^{2}+\cdots
$$

If $\xi=\frac{-(\sqrt{\beta}-2) c_{1} f\left(x_{m}\right)\left(f^{\prime}\left(x_{m}\right)\right)^{2}}{(1-\sqrt{\beta})\left(f\left(x_{m}\right)\left(f^{\prime}\left(x_{m}\right)\right)^{2}-\left(f\left(x_{m}\right)\right)^{2} f^{\prime \prime}\left(x_{m}\right)\right)}$, BMM11 reduces to the following third-order convergent iterative algorithm,

$$
x_{m+1}=x_{m}-\frac{\beta \sqrt{\beta}\left(f\left(x_{m}\right)\right)^{2} f^{\prime \prime}\left(x_{m}\right)+(\beta+\sqrt{\beta}-\beta \sqrt{\beta}) f\left(x_{m}\right)\left(f^{\prime}\left(x_{m}\right)\right)^{2}+\left(\frac{(\sqrt{\beta}-2) c_{1}}{1-\sqrt{\beta}}\right) \sqrt{\beta}\left(f\left(x_{m}\right)\right)^{2} f^{\prime}\left(x_{m}\right)}{\left(f^{\prime}\left(x_{m}\right)\right)^{3}+\left(\frac{(\sqrt{\beta}-2) c_{1}}{1-\sqrt{\beta}}\right) f\left(x_{m}\right)\left(f^{\prime}\left(x_{m}\right)\right)^{2}},
$$

then which has has error equation as

$$
e_{m+1}=\frac{\left[\beta(5 \beta+5)-\sqrt{\beta}\left(\beta^{2}+7 \beta+3\right)+1\right] c_{1}^{2}+\left[\sqrt{\beta}\left(14 \beta+2 \beta^{2}\right)-\beta(10 \beta+6)\right] c_{2}}{\beta \sqrt{\beta}(\sqrt{\beta}-1)^{2}} e_{m}^{3}+\cdots
$$

\section{$3 \quad$ Numerical Results}

In this section, the proposed iterative methods BMM1 to MM11 given by (5), (6), (11), (12), (13), (14), (15), (38), (39), (41) and (42), respectively, are used to approximate real multiple roots for a nonlinear equation and the calculations are done by using Maple 16. We used the test functions which are displayed in Table 1, and obtained the approximate zero $\alpha$, with a maximum number iterations 3. In Table 2, "- "means that the iterative method fails to converge to the multiple root. From Table 2, we can see that, for the given test functions and initial guesses, the difference between the root $\alpha$ and the approximation $x_{m}$ for test functions are displayed. Also we compare the values of $\left|x_{3}-\alpha\right|$ for the proposed iterative methods and the three methods in [11]. The efficiency of the our proposed method are evaluated by the concept of efficiency index which is defined as $E F F=p^{\frac{1}{d}}$, where the value of $p$ is the convergence order of the method and $d$ is the number of functions and derivatives evaluations per step. In Table 3, we compare EFF of BMM1 to BMM7 and other methods in [3, $5,6,7,9,13,16]$ whose the multiplicity of the root of nonlinear equation is single multiplicity. We enumerate the methods 
according to decreasing order of efficiency index and multiplicity $\beta$ of roots for all our proposed methods. Also, we employ the presented algorithms BMM8 to BMM11, solve some nonlinear equations with multiplicity $\beta$. The following stopping criteria are used to computer programs is $\left|x_{m}-\alpha\right|<\epsilon$, where $\epsilon=10^{-16}$, then we use Tables 5 to 6 , show the number of iterations with $\xi=0.5$ and $\xi=1.0$. In these tables, we compare the results by the modified Newton's method (NM) [14], the Euler-Chebyshev method (ECM) [15], Hally-like method (HM) [9], Osada's method (OM) $[13,22]$ and our methods BMM8 to BMM11.

Table 1: The functions and their roots $\alpha$

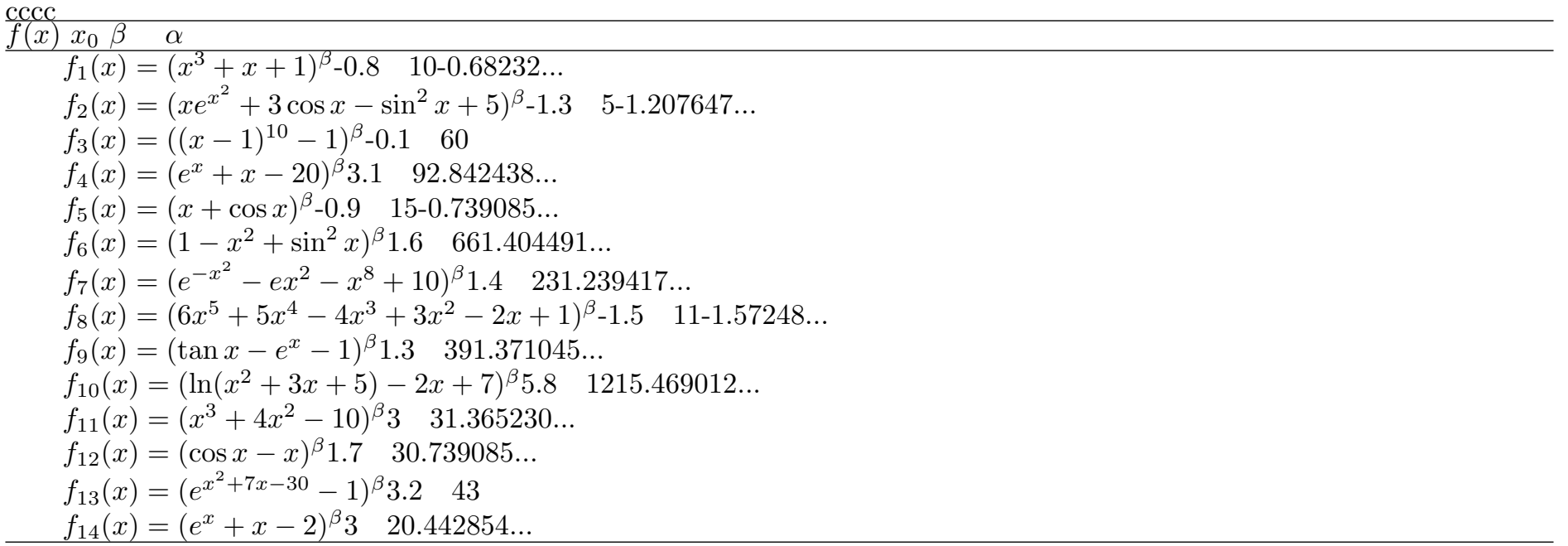

Table 2: Comparison of the values $\left|x_{3}-\alpha\right|$ for the various iterative methods

\begin{tabular}{|c|c|c|c|c|c|c|c|c|c|c|}
\hline$f(x)$ & BMM1 & BMM2 & BMM3 & BMM4 & BMM5 & BMM6 & BMM7 & $\mathrm{NM}[20]$ & $\mathrm{OM}[20]$ & $\mathrm{EC}$ \\
\hline$f_{1}$ & $0.4 \times 10^{-64}$ & $0.3 \times 10^{-245}$ & $0.7 \times 10^{-786}$ & $0.2 \times 10^{-833}$ & $0.2 \times 10^{-804}$ & $0.3 \times 10^{-841}$ & $0.4 \times 10^{-810}$ & $0.2 \times 10^{-614}$ & $0.1 \times 10^{-776}$ & 0.5 \\
\hline$f_{2}$ & $0.7 \times 10^{-52}$ & $0.3 \times 10^{-253}$ & $0.9 \times 10^{-565}$ & $0.8 \times 10^{-835}$ & $0.7 \times 10^{-829}$ & $0.4 \times 10^{-867}$ & $0.1 \times 10^{-843}$ & $0.2 \times 10^{-443}$ & $0.1 \times 10^{-563}$ & 0 . \\
\hline$f_{3}$ & $0.1 \times 10^{-26}$ & $0.5 \times 10^{-115}$ & $0.2 \times 10^{-295}$ & $0.7 \times 10^{-387}$ & $0.2 \times 10^{-364}$ & $0.4 \times 10^{-395}$ & $0.6 \times 10^{-374}$ & - & - & 一 \\
\hline$f_{4}$ & $0.5 \times 10^{-58}$ & $0.3 \times 10^{-241}$ & $0.1 \times 10^{-676}$ & $0.4 \times 10^{-811}$ & $0.3 \times 10^{-792}$ & $0.2 \times 10^{-833}$ & $0.4 \times 10^{-799}$ & $0.9 \times 10^{-395}$ & $0.1 \times 10^{-479}$ & 0.3 \\
\hline$f_{5}$ & $0.2 \times 10^{-94}$ & $0.3 \times 10^{-283}$ & $0.6 \times 10^{-1005}$ & $0.4 \times 10^{-956}$ & $0.1 \times 10^{-914}$ & $0.6 \times 10^{-928}$ & $0.2 \times 10^{-916}$ & $0.1 \times 10^{-760}$ & $0.2 \times 10^{-879}$ & 0.1 \\
\hline$f_{6}$ & $0.4 \times 10^{-57}$ & $0.6 \times 10^{-191}$ & $0.7 \times 10^{-985}$ & $0.3 \times 10^{-710}$ & $0.3 \times 10^{-615}$ & $0.6 \times 10^{-645}$ & $0.6 \times 10^{-616}$ & $0.7 \times 10^{-319}$ & $0.8 \times 10^{-371}$ & 0. \\
\hline$f_{7}$ & $0.3 \times 10^{-31}$ & $0.2 \times 10^{-127}$ & $0.2 \times 10^{-366}$ & $0.6 \times 10^{-433}$ & $0.2 \times 10^{-405}$ & $0.1 \times 10^{-449}$ & $0.6 \times 10^{-408}$ & $0.8 \times 10^{-240}$ & $0.1 \times 10^{-301}$ & \\
\hline$f_{8}$ & $0.2 \times 10^{-48}$ & $0.6 \times 10^{-187}$ & $0.3 \times 10^{-637}$ & $0.2 \times 10^{-649}$ & $0.1 \times 10^{-604}$ & $0.1 \times 10^{-636}$ & $0.2 \times 10^{-609}$ & - & - & - \\
\hline$f_{9}$ & $0.3 \times 10^{-25}$ & $0.3 \times 10^{-165}$ & $0.1 \times 10^{-332}$ & $0.2 \times 10^{-540}$ & $0.5 \times 10^{-531}$ & $0.1 \times 10^{-607}$ & $0.7 \times 10^{-533}$ & $0.3 \times 10^{-189}$ & $0.3 \times 10^{-189}$ & 0.9 \\
\hline$f_{10}$ & $0.5 \times 10^{-33}$ & $0.3 \times 10^{-133}$ & $0.3 \times 10^{-399}$ & $0.1 \times 10^{-458}$ & $0.1 \times 10^{-425}$ & $0.2 \times 10^{-472}$ & $0.5 \times 10^{-426}$ & - & - & - \\
\hline
\end{tabular}

Table 3: Comparison of the efficiency index for various iterative methods in the case of single multiplicity ccccc methods Reference $p d \mathrm{EFF}$

Neta (49)[3], $\beta \neq 32.73221 .653$

Neta (51) [3]2.73221.653

Neta and Johnson[16], $\beta=2431.587$

Neta (32)[3] 331.442

Neta $(29)[3], \beta \neq 3331.442$

Halley[9]331.442

BMM3The proposed method, $\beta>1961.442$

BMM4The proposed method, $\beta>1961.442$

BMM5The proposed method, $\beta>1961.442$

BMM6The proposed method, $\beta>1961.442$

BMM7The proposed method, $\beta>1961.442$

Osada[13]331.442

Neta[5]441.414 
BMM2The proposed method, $\beta>1661.348$

Neta[3], $\beta=3231.259$

BMM1The proposed method, $\beta>1461.259$

P.K.Pairda[6]241.190

Boeng In Yun[7]241.190

Table 4: Comparison of the iterations number for various iterative methods in the case $\xi=0.5$

cecceccce

f(x) BMM8BMM9BMM10BMM11NM $[14 \mid$ ECM $[15 \mid$ HM $|9| \mathrm{OM}[13,22$

$f_{11} 33445334$

$f_{12} 43343334$

$f_{13} 43334434$

$f_{14} 33444223$

Table 5: Comparison of the iterations number for various iterative methods in the case $\xi=1$

ccecceccc

$f(x)$ BMM8BMM9BMM10BMM11NM[14]ECM[15]HM[9]OM[13,22

$f_{11} 44345334$

$f_{12} 53343334$

$f_{13} 44446434$

$f_{14} 43434223$

\section{Conclusion}

We introduced a new family of one and two-step iterative methods for finding multiple roots of nonlinear equations. The convergence order for each method is analyzed. Our presented methods BMM1 to BMM7 require two iteration steps to obtain the desired precisions while other methods require more than two steps. The efficiency index of some proposed methods such as BMM3 to BMM7 is 1.442, which is better than the other methods given in Table 3. We applied the variational iteration technique for generating some of one-step iterative methods such as BMM8 to BMM11 for finding the multiple roots of the nonlinear equations; two special cases were also considered. our results may stimulate further research in this field. The interested readers are advised to devote new and innovative applications of the variational iteration methods.

\section{REFERENCES}

[1] W. Bi, H. Ren and Q. Wu, Three-step iterative methods with eighth-order convergence for solving nonlinear equations, J. Comput. Appl. Math. 225, (2009), 105-112.

[2] R. Burden and J. Faires, Numerical Analysis, PWS Publishing Company, Bostan, USA (2001).

[3] B. Neta, New third order nonlinear solvers for multiple roots, Appl. Math. Comput. 202, (2008), 162-170.

[4] B. Neta and A. N. Johnson, High-order nonlinear solver for multiple roots, Comput. Math. Appl. 55, (2008), 2012-2017.

[5] B. Neta, Extension of Murakamis High order nonlinear solver to multiple roots, Int. J. Comput. Math. 87, (2010), 1023-1031.

[6] P. K. Parida and D. K Gupta, An improved method for finding multiple roots and its multiplicity of nonlinear equations in R, Appl. Math. Comput. 202, (2008), 498-503.

[7] B. I. Yun, Transformation methods for finding multiple roots of nonlinear equations, Appl. Math. Comput. 217, (2010), 599-606.

[8] Z. Wu and X. Li, A fourth-order modification of Newton's method for multiple root, IJRRAS. 10(2)(2012), 166-170.

[9] E. Halley, A new exact and easy method for finding the roots of equations generally and without any previous reduction, Phil. Roy. Soc. London. 18, (1964), 136-147.

[10] K. Munish, V. Kanwar and B. Saurabh, On Some Optimal Multiple Root-Finding Methods and their Dynamics, Appl. Appl. Math. 10(1), (2015), 349-367.

[11] R. Thukral, New ninth-order iterative methods for solving nonlinear equations with multiple roots, Amer. J. Comput. Appl. Math. 4(3),(2014), 77-82.

[12] E. Schr Oder, Uber unendlich viele algorithmen zur auflsung der gleichungen, Math. Ann. 2, (1870), 317-365.

[13] Osada, N, An optimal multiple root-finding method of order three, J. Comput. Appl. Math. 51, (1994), 131-133.

[14] C. Chun and B. Neta, A third-order modification of Newton method for multiple roots, Appl. Math. Comput. 211, 
(2009), 474-479.

[15] J. F. Traub, Iterative Methods for the Solution of Equations, Chelsea publishing company, New York (1977).

[16] J. He, Variational iteration method-some recent results and new interpretations, J. Comput. Appl. Math., 207, (2007), 3-17.

[17] H.H.H. Homeier, On Newton-type methods for multiple roots with cubic convergence, J. Comput. Appl. Math. 231, (2009), 249-254.

[18] K. Noor, M. Noor and S. Momani, Modified Householder iterative methods for nonlinear equations, Appl. Math. Comput. 190, (2007), 1534-1539.

[19] M. Noor, New classes of iterative methods for nonlinear equations, Appl. Math. Comput. 191, (2007), 128-131.

[20] M. Noor and F. Shah, Variational iteration technique for solving nonlinear equations, J. Appl. Math. Comput. 31, (2009), 247-254.

[21] M. Noor, F. Shah, K. Noor and E. Al-said, Scientific Research and Essays, 6, (2011), 1344-1350.

[22] N. Osada, Improving the order of convergence of iterative functions, J. Comput. Appl. Math. 98, (1998), 311-315. 